\title{
Meaningfulness and Trigram Recognition ${ }^{1}$
}

\author{
Edwin Martin and Arthur W. Melton \\ University of Michigan, Ann Arbor, Michigan 48104
}

\begin{abstract}
Recognition, after $1,3,6,15$, and 30 intervening events, for CCC and CVC trigrams of low, medium, and high meaningfulness (M) was studied in the Shepard-Teghtsoonian paradigm. Correct recognition varied directly with $M$ and inversely with number of intervening events. False recognition varied inversely with $M$ and increased with total number of presentations. Confidence in correct recognitions varied directly with $\mathbf{M}$ and inversely with number of intervening events, but remained stable over the experimental session. Confidence in "new" judgments of new trigrams declined sharply over the experimental session. Evidence is presented to the effect that false recognition is largely item specific and not a matter of general decision criterion.
\end{abstract}

Shepard (1963) has argued that a characteristic of verbal units low in meaningfulness $(M)$ is that they are sequentially unintegrated. Of the two trigrams RZQ and MOP, the former may be viewed as more fractionable than the latter from the point of view of $S$ s who are faced either with reproducing it in recall or with using it as a stimulus in paired-associate learning.

Melton's (1963) intra-unit interference interpretation of forgetting in the short-term memory situation is consistent with Shepard's interpretation of $\mathrm{M}$. A low-M unit is seen to be received in memory as a set of more or less independent elements that in recall must be assembled in the presence of interelement interference. A high-M unit, however, is seen to be received as approximately a single element without internal interference. This interpretation is supported by Murdock's (1961) finding that short-term recall of word

\footnotetext{
1 This research was supported by the Advanced Research Projects Agency, Department of Defense, monitored by the Air Force Office of Scientific Research, under Contract Nos. AF 49(638)-1235 and AF 49(638)-1736 with the Human Performance Center, Department of Psychology. The authors acknowledge their debt to Roger B. Howard for preparation of the stimulus materials, J. Kirby Thomas for data collection, and Gerald M. Reicher for programming and computing.
}

triads closely approximates short-term recall of consonant trigrams, and by Peterson, Peterson, and Miller's (1961) finding that low-M CVC trigrams are more poorly recalled than high-M CVC trigrams. The BrownPeterson type of short-term memory test used in these studies requires sufficient integration of the to-be-recalled item to permit verbal production. Such required integration is also the characteristic of response learning in the paired-associate situation. The positive relation between $M$ and ease of the responselearning phase in the paired-associate task (e.g., Underwood \& Schulz, 1960, p. 35f, 92f) thus lends further generality to Shepard's interpretation of the $M$ variable.

Martin's (1968) analysis of the pairedassociate situation in terms of variable stimulus encoding is also consistent with Shepard's interpretation of M. Low M permits, owing to fractional representation, more variable functional encoding of stimuli than does high $\mathrm{M}$, to the end that paired-associate learning is impeded with low-M stimuli. That stimulus $M$ produces this effect is well documented (e.g., Goss \& Nodine, 1965, Exp. 7). Moreover, given that $M$ and serial integration covary directly, it follows that stimulus recoding in the second task in interference transfer paradigms should be 
more likely with low-M than with high-M stimuli, to the end that more retroactive interference should be observed when the stimuli are high $\mathrm{M}$ than when they are low $\mathrm{M}$. This too has been documented (e.g., Bryk \& Kausler, 1966).

Extension of this view of how $M$ affects recall, response learning, and stimulus selection to how $M$ might affect recognition is somewhat complicated. It is clear that the false-recognition (false-positive) rate should vary inversely with $\mathbf{M}$. Given that upon presentation of a trigram that trigram is entered into memory either fractionately or unitarily according to its $\mathrm{M}$ value, and given that other trigrams of the same general $M$ level are also entered into memory, it follows that memory will be more cluttered with nonunique fragments when the trigrams are low than when they are high $\mathrm{M}$. If $S$ is under instruction to indicate (recognize) second occurrences of previously presented trigrams, it must be that he will more often mistakenly recognize new low-M trigrams than new high-M trigrams. But how he will respond to de facto second occurrences is not clear. It does not follow necessarily that high-M trigrams will be better recognized than low-M trigrams. This is because observed correct recognitions have two potential sources: true recognitions and false-positive recognitions. While the latter component is expected to vary inversely with $M$, the former may vary with $\mathrm{M}$ either directly or not at all. If $S$ requires a complete, flawless match between the currently presented trigram and something in memory, then a direct relation between $\mathbf{M}$ and true recognition must be predicted. As the match criterion is lowered, this relation should steadily deteriorate until no relation obtains. At some point, the direct relation between $M$ and true recognition should exactly cancel the inverse relation between $M$ and false-positive recognition.

The experiment to be reported may be viewed as a systematic exploration of the role of $M$ in a recognition task. Such a study seems a prerequisite to further theorizing on the relations among recall, response learning, stimulus selection, and recognition. The need for empirical determination is emphasized by two recent theoretical formulations: (a) Peterson (1967) and Melton (1967) have discussed the merits of a mediative editing mechanism in recall situations. The most likely candidate for this mechanism is some sort of recognition process. (b) On previous occasions it has been argued that association activation depends directly on the probability of stimulus identification, that stimulus identification depends directly upon stimulus $M$, and that stimulus identification is at bottom stimulus recognition (Höffding, 1891; Martin, 1968; Rock, 1962).

\section{METHOD \\ Design}

The recognition paradigm used was that of Shepard and Teghtsoonian (1961). Over a sequence of 320 stimulus presentations, 160 different trigrams were presented twice each. The behavior under study was $S$ 's ability to recognize a trigram on its second occurrence. The number of other trigrams intervening between the two occurrences was either $1,3,6,15$, or 30. All 160 trigrams were either CCCs or CVCs and either of low, medium, or high meaningfulness (M). Thus the number of intervening trigrams was a within$S$ s variable, while the type of trigram and level of $\mathbf{M}$ were both between-Ss variables.

\section{Materials}

The CCCs were drawn from Witmer (1935), the CVCs from Archer (1960). What follows is the mean $M$ value and, in parentheses, the range of values for the 160 trigrams used in each of the six subexperiments (two trigram types, three $M$ levels): CCCs-18.7 (0-25), 46.0 (42-58), and 77.1 (71-96), CVCs-16.8 (2-26), 49.1 (42-58), and $89.6(78-100)$, respectively, for low, medium, and high $\mathrm{M}$.

Given that a list of 160 trigrams was required for each of three levels of $\mathrm{M}$, it follows that complete control of intralist similarity was impossible. However, post hoc statistics can be figured. Thus, with respect to the 18 consonants that could appear in the first position, the frequencies of repetition ranged from 7 to 11 for low-M CCCs, 8-10 for medium-M CCCs, and 3-13 for high-M CCCs. The corresponding ranges for the CVCs are $2-38,0-13$, and $0-11$, respectively. The 
enormous range for low-M CVCs is due to 38 trigrams that began with $\mathrm{X}$. Among the medium- and high-M CVCs, none began with $X$. The following are the standard deviations $(S D \mathrm{~s})$ of the frequencies of repetition of the Position-1 consonants: CCCs-1.24, .74, and $2.73, \mathrm{CVCs}-9.41,2.98$, and 3.15 , respectively, for low, medium, and high $\mathrm{M}$. The greater is the $S D$ the more were a given several of the consonants used repeatedly.

A similar frequency count for reusage of Position 1-2 bigrams can be figured. For the six experimental conditions, the following numbers of different bigrams occurred in the 1-2 position: CCCs-117, 127, and 110 , CVCs $-65,75$, and 70 , respectively, for low, medium, and high M, out of a possible 160. Further statistics on Position 1-2 bigram frequencies are given in Table 1. The top row gives the number of different bigrams that occurred more than once. The second and third rows list the mean and $S D$ of frequency of repetition of bigrams, including those bigrams that occurred only once. in intralist similarity, if favors confusion among higher-M CVCs.

Two 35-mm slides were made of each of the 160 trigrams of a given combination of trigram type and $M$ level. The resulting slides were then arranged into a presentation order such that each number of intervening events $(1,3,6,15,30)$ was represented 32 times; that is, of the 160 double occurrences, 32 had one intervening presentation, 32 had three intervening presentations, and so on. The presentation order had three additional properties: (a) The probability of a new trigram following immediately after a new trigram was .24; or an old following an old, .23; of an old following a new, .26; and of a new following an old, .27. Moreover, these dyads were evenly distributed over the sequence of presentations. (b) The recurrence of initial letters was distributed approximately uniformly over the experimental session. (c) Each of the five lag (number of intervening events) conditions occurred equally often (eight times) in each quarter of the experimental sequence of events.

TABLE 1

Position 1-2 Bigram Frequency Data

\begin{tabular}{|c|c|c|c|c|c|c|}
\hline & \multicolumn{3}{|c|}{$\mathrm{CCC}$} & \multicolumn{3}{|c|}{$\mathrm{CVC}$} \\
\hline & Low & Medium & High & Low & Medium & High \\
\hline Number repeated bigrams & 32 & 31 & 38 & 35 & 47 & 54 \\
\hline Mean repetitions per bigram ${ }^{a}$ & 1.37 & 1.26 & 1.45 & 2.46 & 2.13 & 2.29 \\
\hline SD repetitions per bigram ${ }^{a}$ & .67 & .74 & 1.23 & 2.86 & 1.23 & .99 \\
\hline
\end{tabular}

${ }^{a}$ Includes bigrams used only once.

When all three letter positions are taken into account, each of the 160 trigrams within a given list was unique.

These intralist-similarity statistics may be summarized as follows: Over the three levels of M for CCCs, there is no variable problem of intralist item similarity of any account. For CVCs, $41 \%$ fewer different bigrams occur in Position 1-2 than for CCCs, indicating that among CVCs there is considerably greater formal intralist similarity than among CCCs. This is due primarily to the limited number of vowels that can occur in the second position. Over the three levels of $\mathrm{M}$ for CVCs, the mean frequency of bigram repetition remains approximately constant $(2.46,2.13,2.29)$, although the number of different repeated bigrams increases $(35,47,54)$. This adds up to a complicated picture of intralist similarity for CVCs that, in net, spells increasing intralist similarity with increasing $M$ level. Thus if there is any experimentally effective trend
There was one order of events as defined by the five lag conditions. But within each of the six subexperiments (two trigram types, three levels of $M$ ), there were five different sequences of trigrams so that each trigram was assigned equally often to each lag condition.

\section{Subjects}

The $S$ s were 120 University of Michigan undergraduates who volunteered for paid (\$1.25) participation. Of these, 20 were assigned to each of the six combinations of trigram type and $\mathrm{M}$ level. The $S \mathrm{~s}$ were run in groups of 2-5. No experimental combination was assigned an $n$th group until all the other combinations had been assigned $\boldsymbol{n}-1$ groups. Each successive subgroup of $S \mathrm{~s}$ in a given combination was tested on the next one of the five trigram presentation orders. 


\section{Procedure}

A group of 2-5 Ss was seated in classroom deskchairs. On the arm of each chair was a button panel with six buttons. The three buttons to the left were labelled "new", the three to the right, "old." Approximately $1.8 \mathrm{~m}$. in front of the $S \mathrm{~s}$ there was a screen onto which the trigrams were projected by a Carousel projector. The $S$ s were told that upon presentation of a trigram, they were to decide whether it be new or old. The outermost button was to be pressed were they highly confident (certain) of the correctness of their decision, the innermost if they had little or no confidence. For analytic purposes, the buttons may be imagined numbered 1 through 6 from left to right; thus Buttons 1-3 stand for "new," 4-6 for "old." This means that were $S$ highly confident that a particular trigram be old, he presumably would press Button 6; if he were practically guessing, Button 4.

For each trigram presentation, the following sequence of events occurred: onset of slide projection; $3.5 \mathrm{sec}$. later, onset of an overhead warning light to signal the $S$ s that they had remaining $1 \mathrm{sec}$. to respond; after this 1 sec., a 1.5 -sec. cycling delay occurred, during which the already-made button presses of the $S$ s were punched automatically in paper tape. If $S$ failed to respond by pressing one of the six buttons within the allowed $4.5 \mathrm{sec}$., a red light on his button panel flashed to advise him that he should respond faster in the future.

The sequence of 360 presentations was run off without interruption. This, together with instructions, required approximately $45 \mathrm{~min}$.

\section{RESULTS}

The number of failures to respond within the allowed $4.5 \mathrm{sec}$. was 40,35 , and 87 for the high-, medium-, and low-M CCCs, respectively; and 24, 28, and 23 for the corresponding $\mathrm{M}$ levels of CVCs. These omissions represent $1.7 \%$ of 9600 response opportunities over the combined three M levels of CCCs; .7\% for the CVCs. In the following report of the results, when it is stated that such-and-such proportion of $N$ opportunities resulted in, say, a "new" response, in computation of the proportion the $N$ was actually reduced by the appropriate number of omissions.

\section{Initial Occurrences}

The first time a trigram is presented, it is new de facto; but $S$ s may respond "new" or "old." "Old" responses to new trigrams will be denoted FPs, for false positives. For each of the six combinations of trigram type and $M$ level, the FP rates over the 32 initial occurrences for each number of intervening trigrams are listed in Table 2. Each entry is the proportion of $(20 \mathrm{Ss}) \times(32$ initial occurrences $)=640$ new-trigram stimulus events to which the response "old" (Button 4, 5, or 6) was given (except as $N$ was corrected for failures to respond).

Since the subsequent number of trigrams intervening before the recognition test cannot affect responding on the initial occurrence of a trigram, the FP rates within a given row should not differ among themselves. An inspection of

TABLE 2

Proportion false Posttives ( $F P$ ) and Proportion Correct Recognitions $(P$ )

\begin{tabular}{|c|c|c|c|c|c|c|c|c|c|c|}
\hline \multirow{3}{*}{$\begin{array}{l}\text { Type } \\
\qquad \begin{array}{c}\text { M } \\
\text { Level }\end{array}\end{array}$} & \multicolumn{10}{|c|}{ Number of intervening trigrams } \\
\hline & \multicolumn{2}{|c|}{1} & \multicolumn{2}{|c|}{3} & \multicolumn{2}{|c|}{6} & \multicolumn{2}{|c|}{15} & \multicolumn{2}{|c|}{30} \\
\hline & FP & $\mathbf{P}$ & FP & $\mathrm{P}$ & FP & $\mathbf{P}$ & FP & $\mathbf{P}$ & $\mathrm{FP}$ & $\mathbf{P}$ \\
\hline \multicolumn{11}{|l|}{$\mathrm{CCC}$} \\
\hline High & .234 & .954 & .233 & .932 & .231 & .935 & .198 & .913 & .207 & .803 \\
\hline Medium & .355 & .942 & .400 & .915 & .374 & .887 & .368 & .826 & .380 & .708 \\
\hline Low & .413 & .941 & .402 & .882 & .391 & .842 & .370 & .803 & .395 & .708 \\
\hline \multicolumn{11}{|l|}{ CVC } \\
\hline High & .170 & .991 & .201 & .983 & .206 & .983 & .171 & .948 & .184 & .905 \\
\hline Medium & .306 & .976 & .294 & .989 & .301 & .969 & .254 & .935 & .317 & .823 \\
\hline Low & .400 & .969 & .430 & .953 & .429 & .934 & .421 & .871 & .404 & .770 \\
\hline
\end{tabular}


Table 2 reveals no trends in FP rates over the number of intervening items. Collapsing over number of intervening trigrams, the FP rates are: CCCs- $-394, .375$, and $.220, \mathrm{CVCs}-.417$, .294 , and .186, respectively, for low, medium, and high M. Rate of FPs, then, is strongly related, in an inverse manner, to the $\mathrm{M}$ level of the trigrams presented. For CCCs, the FP rate for low $\mathrm{M}$ is 1.79 times what it is for high $\mathrm{M}$; for CVCs, the FP rate for low M is 2.24 times that for high $\mathrm{M}$. as old. It is interesting that with lesser levels of $\mathrm{M}$, the $\mathrm{FP}$ rate does not stabilize, even with as many as 160 presentations of new trigrams. With greater levels of $M$ (high-M CCCs, medium- and high-M CVCs), however, the FP rates appear to be nearing an asymptote. It is also notweorthy that more of a difference between CCC and CVC stimuli did not materialize. For example, the FP functions for high-M CCCs and high-M CVCs are very much alike, yet approximately $35 \%$ of the

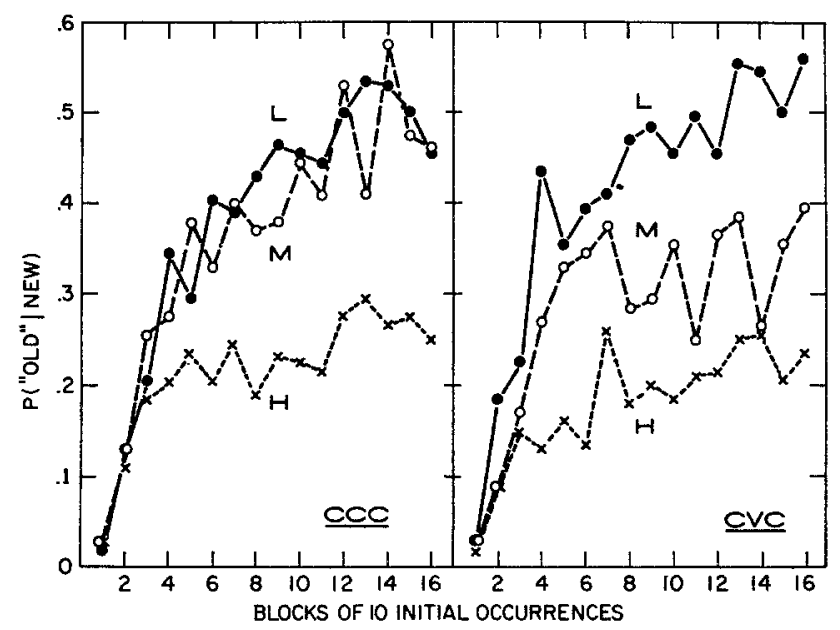

FrG. 1. Proportion "old" responses to new trigrams, as a function of blocks of 10 initial occurrences, for low (L), medium (M), and high (H) $\mathrm{M}$.

The foregoing FP rates are over the 160 initial occurrences of trigrams within each of the six subexperiments (combinations of trigram type and $\mathrm{M}$ level). An examination of successive blocks of ten initial occurrences reveals that FP tendencies began close to zero and increased at rates peculiar to the $M$ level of the trigrams being presented. These functions are shown in Figure 1, where each point is the proportion of $(20 \mathrm{Ss}) \times(10$ occurrences $)=200$ new-trigram stimulus events to which the response "old" was given. Note that the higher the $M$ value, the less likely is $S$ to say "old" to a new trigram; or, conversely, the lower the $M$ value, the more likely is $S$ to identify mistakenly a new trigram
high-M CVCs are ordinary words and all are pronounceable to a reasonable degree. The FP functions for low-M CCCs and low-M CVCs are also very similar, although again there is perforce a clear difference in pronunciability.

Concomitant with the buildup of FPs shown in Figure 1 is a deterioration in confidence. At the outset, $S$ s have high confidence in the correctness of "new" responses to new trigrams; but as the sequence of presentations advances, confidence declines at a rate inversely related to $M$ level. For example, consider the two extreme conditions. For low-M CCCs, the proportion responses to Buttons 1, 2, and 3 over the first 30 initial 
occurrences are $.781, .154$, and .065 , respectively; while the corresponding proportions over the last 30 initial occurrences are .256 , .372 , and .375 . In other words, the J-shaped distribution of choices over the three "new" buttons evident early in the sequence shifts to an approximately uniform distribution later in the sequence. In comparison, the proportion-responses distribution over Buttons 1, 2, and 3 for high-M CVCs greatly resists shifting toward uniformity. The appropriate figures are $.899, .095$, and .006 for the first 30 initial occurrences; $.679, .206$, and .115 for the last 30 initial occurrences.
As to the possibility of having attained a steady state with respect to confidence in "new" responses to new trigrams, one can say from Figure 2 that there is only a suggestion that asymptotic confidence is being approached, and then only for the higher-M materials.

\section{Second Occurrences}

The second time a trigram is presented, it is, of course, old in fact; but the likelihood of $S$ responding "old" to that trigram is dependent jointly on the $M$ value of the trigrams being presented and the number of trigrams that intervene between initial and test occurrences.

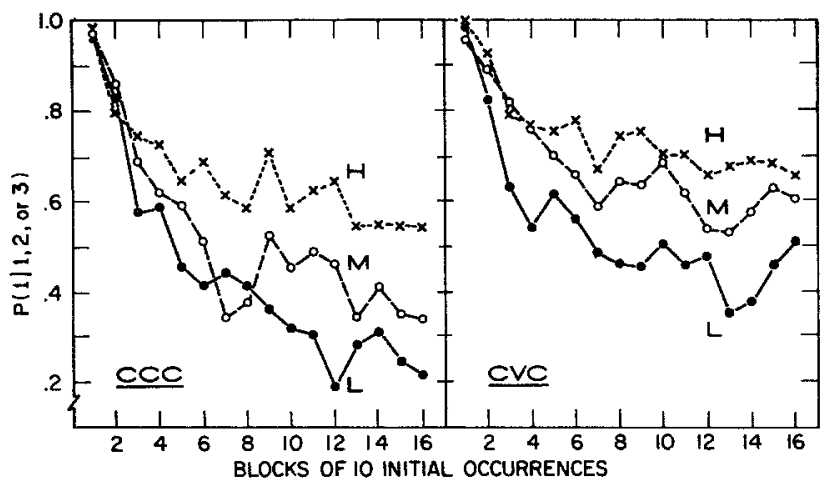

FIG. 2. Proportion Button-1 responses given Button 1, 2, or 3, as a function of blocks of 10 initial occurrences, for low (L), medium $(\mathrm{M})$, and high $(\mathrm{H}) \mathrm{M}$.

The rather dramatic decline in confidence in "new" responses to new trigrams is shown in Figure 2, where the proportion Button-1 responses ("Certain it is new"), given that either Button 1 or 2 or 3 was pressed (that, is given a "new" response), is plotted against successive blocks of ten initial occurrences. As with FP rates, level of $M$ induces reliable differences: $S$ s are more often certain that a new trigram is new when the trigram is high $M$ than when it is low $M$. Unlike FP rates, however, there is a clear difference in confidence traceable to type of material: $S$ s are consistently more confident in their "new" responses when the trigrams are CVCs as compared to when they are CCCs.
For a given combination of trigram type, $\mathrm{M}$ level, and number of intervening trigrams, let $P$ be the proportion of $(20 \mathrm{Ss}) \times(32$ second occurrences $)=640$ old-trigram stimulus events to which the response "old" (Button 4, 5 , or 6) was given. For each of the six experimental combinations of trigram type and $M$ level, the $P$ value for each repetition lag (number of intervening trigrams) is presented in Table 2. These data clearly indicate that recognition has a direct dependency on $M$ level, that recognition declines steadily with increasing number of intervening events, and that recognition is better for CVCs than for CCCs.

It is critical for later discussion to note that 
proportion "old" responses to second (or test) occurrences was higher given that "old" (a FP) was the response to the initial occurrence than was the case when "new" was the response to the initial occurrence. Moreover, the magnitude of this difference is inversely related to $\mathrm{M}$. Consider the extreme conditions, collapsed over number of intervening trigrams: For high-M CVCs, if the initial occurrence was falsely recognized $\left(\mathrm{O}_{1}\right)$, the proportion recognitions on test occurrences is .978 ; whereas if the initial occurrence was correctly called new $\left(\mathrm{N}_{1}\right)$, the proportion recognitions is .958 . The corresponding proportions for low-M CCCs are .900 and .792 . The appropriate proportions, collapsed over number of intervening presentations, for all six subexperiments (type of trigram, level of $\mathrm{M}$ ) are given in Table 3.

\section{TABLE 3}

Proportion "OlD" RESPONSES TO Old Trigrams ACCORding to WHETHER $S$ RESPONDED "OlD" $\left(\mathrm{O}_{1}\right)$ OR "NEW" $\left(\mathrm{N}_{1}\right)$ ON THE INITIAL OCCURRENCE

\begin{tabular}{ccc}
\hline $\begin{array}{c}\text { Type } \\
\mathbf{M} \\
\text { level }\end{array}$ & $\mathrm{O}_{1}$ & $\mathrm{~N}_{1}$ \\
\hline CCC & & \\
High & .922 & .903 \\
Medium & .908 & .824 \\
Low & .900 & .792 \\
CVC & & \\
High & .978 & .958 \\
Medium & .973 & .924 \\
Low & .934 & .874 \\
\hline
\end{tabular}

Not shown in Table 3 is the further fact that the dependency of proportion "old" responses to a second occurrence on whether the response to the initial occurrence was "old" $\left(\mathrm{O}_{1}\right)$ or "new" $\left(N_{1}\right)$ varies directly with number of intervening presentations. Thus, for example, for low-M CCCs the recognition proportions were .977 given $O_{1}$ and .915 given $N_{1}$ when there was only one intervening presentation, but .803 given $\mathrm{O}_{1}$ and .646 given $\mathrm{N}_{1}$ when there were 30 intervening presentations. In all six experimental combinations (trigram type, $\mathrm{M}$ level), this dependency increased with number of intervening presentations.

As with initial occurrences, confidence in correct-recognition responding to second occurrences was materially affected by the experimental variables manipulated. Consider again the extreme cases. For low-M CCCs, the distribution of responses to Buttons 4,5 , and 6 is given by the proportions $.058, .134$, and .808 , respectively, with one intervening trigram; however, for 30 intervening trigrams, the corresponding proportions are $.134, .320$, and .546. Thus as the recognition test is delayed, confidence in correct recognitions declines. For high-M CVCs, the loss in confidence is less dramatic: the proportion responses to Buttons 4,5 , and 6 shift from $.003, .024$, and .973 to. $038, .108$, and .853 , respectively. Also evident in the foregoing sets of proportions is the difference in confidence in correct-recognition responses between levels of $M$.

In contrast to the case for initial occurrences, however, confidence in correct-recognition responses did not decline as the experimental session advanced. What follows is proportion Button-6 responses ("Certain it is old") given that either Button 4, 5, or 6 was pressed (that is, given an "old" response), collapsed over the entire experimental session, as well as over number of intervening presentations. With each of these proportions, in parentheses, is the $S D$ of the proportion Button- 6 responses as that proportion varied about the given overall proportion in successive blocks of 10 recognition tests. CCCs-.697 (.046), .714 (.056), and .880(.058) for low, medium, and high M, respectively; CVCs-.808 (.046), .902 (.042), and $.925(.035)$, respectively. Thus confidence in correct "old" responses is clearly affected by $M$ level and trigram type, but varies hardly at all as the experimental session advances. 


\section{Discussion}

The results may be summarised as follows. Recognition is directly related to the $M$ level of the trigrams presented and declines as the number of intervening presentations increases. False recognition is inversely related to $M$ level and increases with the total number of presentations experienced. Confidence in the correctness of "old" responses to old trigrams is lower the longer the delay of the recognition test and the lower the $\mathrm{M}$ level of the trigrams presented; but this confidence does not decline as the experimental session advances. Confidence in "new" responses to new trigrams declines with number of presentations experienced and is lower for lower- $\mathrm{M}$ trigrams. Which response, "old" or "new", is given to the initial occurrence of a trigram is a determiner of the likelihood of correct recognition at the time of the second, or test, occurrence.

Several features of the present results bear comparison with earlier recognition results by Shepard and Teghtsoonian (1961) and by Melton, Samerof, and Schubot (1967). In both of these experiments, the items presented were three-digit numbers. Proportion recognitions under a 6 -sec. rate of presentation (the same rate used here) declined over number of intervening presentations at a rate that most closely approximates that of the low- and medium-M CCCs of the present experiment. The proportion FPs as a function of number of preceding presentations in the Melton et al. experiment, however, levelled off at between .30 and .35 , and did so in the neighborhood of 60 prior presentations. In the Shepard-Teghtsoonian experiment, proportion FPs continued to rise throughout to a value of about .28 (200 presentations) with no indication of an asymptote. In Figure 1 in the present report, no asymptote is apparent for FPs in the low- and medium-M CCC subexperiments, and the terminal FP rate is in the neighborhood of .50 ( 360 presentations). Thus generalization about FP rates and their approach to a steady state is best deferred.
The present finding that recognition on the second, or test, occurrence depends to a measurable extent upon whether an "old" or a "new" response was given on the initial occurrence corroborates the same effect reported by Melton et al. The superiority of recognition given an "old" response to the initial occurrence $\left(\mathrm{O}_{1}\right)$ over that given a "new" response to the initial occurrence $\left(\mathrm{N}_{1}\right)$, as observed in the low-M CCC subexperiment, is illustrated in Figure 3, along with the corresponding result from the Melton et al. experiment (Experiment I). The import of this phenomenon is that it indicates rather strongly that FP tendencies are largely item specific and not simply a matter of a general decision criterion. In other words, whatever the basis for false recognition on an initial occurrence. it is identifiable as a contributor to the likelihood of emitting an "old" response on the second occurrence of that trigram. The type result shown in Figure 3 is not explainable in terms of an indiscriminate response bias determined by the average confusability among the items being presented; for if this were the case, the $\mathrm{O}_{1}$ and $\mathrm{N}_{1}$ functions would be

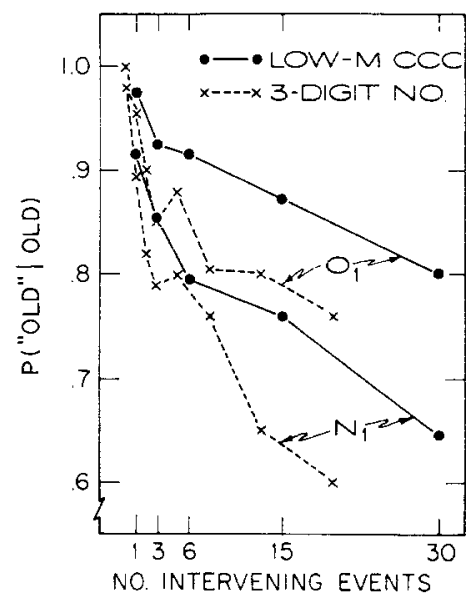

FIG. 3. Proportion "old" responses to old low-M CCCs (solid) and to old three-digit numbers (dashed) where "old" $\left(\mathrm{O}_{1}\right)$ and "new" $\left(\mathrm{N}_{1}\right)$ responses were given to initial occurrence, as a function of number of intervening presentations. (Three-digit-number data from Melton et al., 1967). 
indistinguishable. This conclusion that FP tendency is to some degree item specific is critical to our contention that $M$ exerts its effect in various verbal tasks in the manner suggested by Shepard (1963), namely, via the fractionative characteristics of the items in question.

There is an alternative interpretation of the results of the foregoing analysis. Kintsch (1967) has proposed that verbal units differ at the time of their initial occurrence in a priori familiarity, and that depending on whether they are correctly accepted as new or falsely accepted as old, they are assigned new (hypothetical) familiarity values so that, statistically, units initially more familiar remain more familiar. This statement hardly does justice to Kintsch's parametric model, but it is sufficient to point out that his formulation predicts that trigrams called "old" on their initial presentation are more likely to be called "old" on their second presentation than are trigrams initially called "new." Although Kintsch's model seems to deal with psychological familiarity, none of his parameters can be uniquely identified with familiarity. We prefer the serialintegration interpretation because it appears to be more frequently implicated in a great variety of verbal tasks. In any case, both interpretations are item specific.

As argued at the outset, Shepard's (1963) hypothesis about $M$ permits the prediction that FP rate will vary inversely with $M$. This prediction was clearly confirmed (Figure 1).
However, it did not seem to follow necessarily that high-M trigrams will be better recognized then low-M trigrams. As stated earlier, observed correct recognitions have true recognition and false-positive recognition as possible sources. In order to estimate true recognition, let $P^{\prime}$ be the probability of a bona fide recognition, $P$ the observed proportion recognitions, and $F P$ the observed proportion false positives. The recognition of an old trigram will occur by true recognition with probability $P^{\prime}$ or by false-positive recognition $\left(1-P^{\prime}\right) F P$ proportion of the time. If we solve the equation $P=P^{\prime}+(1-$ $\left.P^{\prime}\right) F P$ for $P^{\prime}$ we obtain

$$
P^{\prime}=\frac{P-F P}{1-F P} .
$$

Each of the 30 pairs of $P$ and $F P$ values in Table 2 was substituted into this formula and the resulting $P^{\prime}$ values plotted in Figure 4, where it is evident that trigram recognition remains a definitive function of $M$ after correction for $F P$ tendencies. This means that in this particular recognition task, there is a direct relation between $M$ and true recognition that exceeds the counter relation between $M$ and false-positive recognition.

The results shown in Figure 4 must be considered with some reservation, however. As pointed out earlier, $F P$ rates for low- and medium-M CCCs and for low-M CVCs clearly did not stabilize at an asymptote (Figure 1). Ideally, estimates of $P^{\prime}$ should be

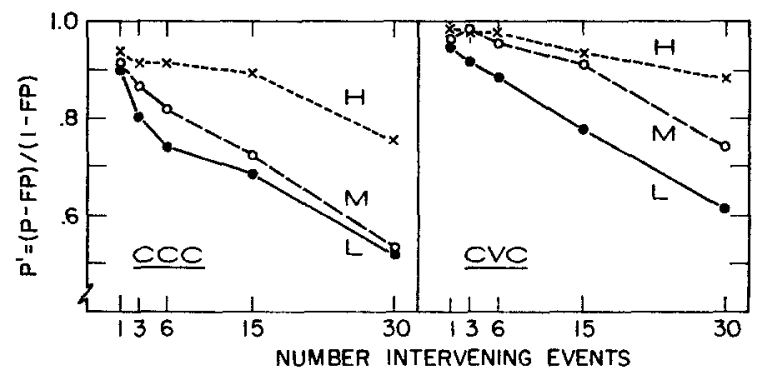

FIG. 4. Proportion "old" responses to old trigrams, corrected for FP rate, as a function of number of intervening events, for low (L), medium (M), and high (H) $M$. 
made only after a steady state of $F P$ responding has been achieved. Nevertheless, approximate steady states for FPs were achieved for highand medium-M CVCs and differences in $P^{\prime}$ values were observed, with high-M CVCs being better recognized than medium-M CVCs.

The conclusion that $M$ affects correct recognition in the same way that it affects recall does not necessarily mean, as Postman, Jenkins, and Postman (1948) and many others believe, that recall and recognition depend on the same underlying processes. To establish process correspondence, one must proceed further by somehow defining and explicating in the recall situation the type of performance in the recognition situation identified as false-positive responding. To our knowledge, the extent of associative construction in recall, which qualifies as a false-positive analogue, has not been considered either conceptually or experimentally. Although some thought has been given to implicit rejection of otherwise correct recalls in terms of a mediative editing mechanism (Peterson, 1967), experimental isolation of this process has not yet been attained.

Finally, the present results serve to delineate the role of $M$ in stimulus identification: Low-M verbal units are both less positively identifiable and more mistakenly identifiable, and hence are a less reliable nominal source of functional stimulation that are high-M verbal units.

\section{REFERENCES}

ARCHER, E. J. A re-evaluation of the meaningfulness of all possible CVC trigrams. Psychological Monographs, 1960, 74, No. 10 (Whole No. 497).

BryK, J. A., \& Kausler, D. H. Stimulus meaningfulness and unlearning in the A-B, A-C transfer paradigm. Journal of Experimental Psychology, 1966, 71, 917-920.

Goss, A. E., \& Nodine, C. F. Paired-associate learning. New York: Academic Press, 1965.
HöFfDING, H. Outlines of psychology. (Transl. by M. E. Lowndes) London: Macmillan, 1891.

KINTSCH, W. Memory and decision aspects of recognition learning. Psychological Review, 1967, 74, 496-504.

Martin, E. Stimulus meaningfulness and pairedassociate transfer: An encoding variability hypothesis. Psychological Review, 1968, 75, 421-441.

Melton, A. W. Implications of short-term memory for a general theory of memory. Journal of Verbal Learning and Verbal Behavior, 1963, 2, 2-21.

Melton, A. W. Decision processes in retrieval from memory. In B. Kleinmuntz (Ed.), Concepts and the structure of memory. New York: Wiley, 1967. Pp. 215-225.

Melton, A. W., Samerof, A., \& Schubot, E. D. Shortterm recognition memory. Human Performance Center Memorandum Report No. 2, University of Michigan, 1967.

MURDock, B. B., JR. The retention of individual items. Journal of Experimental Psychology, 1961, 62, 618-625.

Peterson, L. R. Search and judgment in memory. In B. Kleinmuntz (Ed.), Concepts and the structure of memory. New York: Wiley, 1967. Pp. 153180.

Peterson, L. R., Peterson, M. J., \& Miller, A. Short-term retention and meaningfulness. Canadian Journal of Psychology, 1961, 15, 143-147.

Postman, L., Jenkins, W. O., \& Postman, D. L. An experimental comparison of active recall and recognition. American Journal of Psychology, 1948, 61, 511-519.

Rock, I. A neglected aspect of the problem of recall: The Höffding function. In J. Scher (Ed.), Theories of the mind. New York: Free Press, 1962, Pp. 645-659.

ShePard, R. N. Comments on Professor Underwood's paper. In C. N. Cofer and B. S. Musgrave (Eds.), Verbal behavior andlearning. New York: McGrawHill, 1963. Pp. 48-70.

Shepard, R. N., \& Teghtsoonian, M. Retention of information under conditions approaching a steady state. Journal of Experimental Psychology, 1961, 62, 302-309.

Underwood, B. J., \& Schulz, R. W. Meaningfulness and verbal learning. Philadelphia: Lippincott, 1960.

WITMER, L. R. The association value of three-place consonant syllables. Journal of Genetic Psychology, $1935,47,337-360$.

(Received September 15, 1969) 